\title{
IUDs: Maternity Nurses' Practices of Infection Control Measures
}

\author{
Amera Bekhatroh Rashed ${ }^{1 *}$, Nevin Adel Amer ${ }^{2}$, Zeinab A.Ali ${ }^{3}$
}

${ }^{1,2}$ Nursing department, College of Applied Medical Sciences, Jouf University, Qurrayat, KSA

${ }^{3}$ Physical therapy department, College of Applied Medical Sciences, Jouf University, Qurrayat, KSA

${ }^{3}$ Physical therapy department for surgery, Faculty of physical therapy, Cairo University, Egypt

DOI: $10.36348 /$ sjnhc.2020.v03i11.008 $\quad$ | Received: 09.11.2020 | Accepted: 21.11.2020 | Published: 23.11 .2020

*Corresponding author: Amera Bekhatroh Rashed

\section{Abstract}

Background: The consistent use of recommended infection prevention practices is a critical component of quality health services, as well as a basic right of every patient, client, or staff member in a health care setting. Summary: This review of literature includes three sections. The first section is an overview about IUD; the second section is devoted to infection control measures in family planning unit while the third explain maternity nurses' practice of such measures.

Keywords: This review aims to provide an overview about maternity nurses' practices of infection control measures during insertion and removal of IUDs.

Copyright (C) 2020 The Author(s): This is an open-access article distributed under the terms of the Creative Commons Attribution 4.0 International License (CC BY-NC 4.0) which permits unrestricted use, distribution, and reproduction in any medium for non-commercial use provided the original author and source are credited.

\section{INTRODUCTION}

Intrauterine devices (IUDs) are small devices, often ' $\mathrm{T}$ '-shaped,containing either copper or levonorgestrel, which are inserted into the uterus. They are one form of long-acting reversible contraception which is the most effective types of reversible birth control. Failure rates with the copper IUD is about $0.8 \%$ while the levonorgestrel IUD has a failure rate of $0.2 \%$ in the first year of use. Among types of birth control, they along with birth control implants result in the greatest satisfaction among users [1].

According to Salem 2009, the Egyptian Ebers Papyrus from $1550 \mathrm{BCE}$ and the Kahun Papyrus from 1850 BCE have within them some of the earliest documented descriptions of birth control: the use of honey, acacia leaves and lint to be placed in the vagina to block sperm.

According to Egyptian Demographic Health Survey (EDHS2014), IUDs use rose from a rate of 4 percent in 1980 to a level of 36-37 percent during the 2000-2008 periods. The 2014 EDHS results document that a substantial drop in IUD use took place after 2008, with use of the method decreasing to 30 percent. The decline in IUD use was offset by rises in the use of the pill (from 12 percent in 2008 to 16 percent in 2014) and, to a lesser extent, the injectable (from 7 percent in 2008 to 9 percent in 2014). Also according to EDHS 2014 results ,the highest rate of IUDs use is in Asia
(17.9\% and $27.0 \%$ of women ), followed by Europe (12.4\%; 17.1\%), Africa $(4.4 \% ; 15.4 \%)$, Latin America/Caribbean (7.0\%; 9.6\%), United States (5.3\%), North America $(4.8 \% ; 6.1 \%)$ and Oceania (1.1\%; $1.8 \%)$.

The history of intrauterine devices dates back to the early 1900s. Unlike IUDs, early intrauterine devices crossed both the vagina and the uterus, causing a high rate of pelvic inflammatory disease in a time period when gonorrhea was more common. The first IUD was developed in 1909 by the German physician, Richard Richter of Waldenburg. His device was made of silkworm gut and was not widely used [2].

Ernst Gräfenberg, another German physician after whom the G-spot is named, created the first Ring IUD, Gräfenberg's ring, made of silver filaments. His work was suppressed during the Nazi regime, when contraception was considered a threat to Aryan women. He moved to the United States, where his colleagues H. Hall and M. Stone took up his work after his death and created the stainless steel Hall-Stone Ring. A Japanese doctor named Tenrei Ota also developed silver or gold IUD called the Precea or Pressure Ring. Jack Lippes helped begin the increase of IUD use in the United States in the late 1950s. In this time, thermoplastics, which can bend for insertion and retain their original shape, became the material used for first-generation IUDs. Lippes also devised the addition of the monofilament nylon string, which facilitates IUD 
removal. His trapezoid shape Lippes Loop IUD became one of the most popular first-generation IUDs [3].

The invention of the copper IUD in the 1960s brought with it the capital ' $\mathrm{T}$ ' shaped design used by most modern IUDs. U.S. physician Howard Tatum determined that the ' $\mathrm{T}$ ' shape would work better with the shape of the uterus, which forms a ' $\mathrm{T}$ ' when contracted. He predicted this would reduce rates of IUD expulsion. Together, Tatum and Chilean physician Jaime Zipper discovered that copper could be an effective spermicide and developed the first copper IUD, TCu200. Improvements by Tatum led to the creation of the TCu380A (ParaGard), which is currently the preferred copper IUD. The hormonal IUD was also invented in the 1960s and 1970s; initially the goal was to mitigate the increased menstrual bleeding associated with copper and inert IUDs [4].

\section{MAIN TEXT \\ SECTION I \\ Overview about IUDs}

According to United Nations data on worldwide contraceptive use 2014, IUDs have many types: Nonhormonal copper IUD (ParaGard). IUD with progestogen (Mirena among others).

\section{Mechanism of Action}

All IUDs induce a local inflammatory reaction that disturbs the functioning of the endometrium and myometrium and changes the microenvironment of the uterine cavity. Moreover, these effects alter signaling between uterus and ovary. The entire genital tract seems affected, at least in part because of luminal transmission of fluids accumulating in the uterine lumen. Copper or progesterone-releasing IUDs may attenuate or accentuate the inflammatory response, disturb the physiology of the gametes in the female genital tract, or destroy the viability of the embryos or endometrial receptivity to implantation. Studies on the recovery of eggs reveal that embryos are formed in the tubes of IUD users at a significantly lower rate compared to nonusers. This, rather than the destruction of embryos in the uterus, appears to be the IUD's major mechanism of action [5].

\section{Indications}

The World Health Organization (WHO) and the Centers for Disease Control and Prevention (CDC) 2009 have developed guidelines for practitioners regarding IUD use in a variety of clinical circumstances. IUDs are considered appropriate for the majority of women, including nulliparous women and adolescents. Both immediate postpartum insertion (within 10 minutes of placental delivery) and delayed postpartum insertion (within 4 weeks of placental delivery) are acceptable. Similarly, postabortion (spontaneous or elective) insertion is acceptable.
The World Health Organization (WHO) 2009 also added that IUD use is safe in women with the following conditions:

-History of an ectopic pregnancy, history of pelvic surgery, hypertension or other forms of heart disease, history of deep venous thrombosis, history of migraine headaches, anemia, diabetes or endometriosis.

A copper IUD (shown in Fig. 1) (also intrauterine device, IUD, copper-T, or coil) is a type of intrauterine device. The copper IUD is a type of longacting reversible contraception and is one of the most effective forms of birth control. Copper IUDs are a form of long-acting reversible contraception and are one of the most effective forms of birth control available. The type of frame and amount of copper can affect the effectiveness of different copper IUD models. The failure rates for different models vary between 0.1 and $2.2 \%$ after 1 year of use. The T-shaped models with a surface area of $380 \mathrm{~mm}^{2}$ of copper have the lowest failure rates. The TCu 380A (ParaGard) has a one-year failure rate of $0.8 \%$ and a cumulative 12-year failure rate of $2.2 \%$. Over 12 years of use, the models with less surface area of copper have higher failure rates. The $\mathrm{TCu} 220 \mathrm{~A}$ has a 12 -year failure rate of $5.8 \%$, whereas the $\mathrm{TCu} 380 \mathrm{~A}$ has a 12 -year failure rate of $2.2 \%$. The frameless GyneFix also has a failure rate of less than $1 \%$ per year. Worldwide, older IUD models with lower effectiveness rates are no longer produced [6].

\section{Side effects}

Based on [5] adverse effects of copper IUDs are:

Expulsion: Sometimes the copper IUD can be spontaneously expelled from the uterus. Expulsion rates can range from $2.2 \%$ to $11.4 \%$ of users from the first year to the 10 th year.

Perforation: Very rarely, the IUD can move through the wall of the uterus. Risk of perforation is mostly determined by the skill of the practitioner performing the insertion. For experienced medical practitioners, the risk of perforation is 1 per 1,000 insertions or less.

Infection: The insertion of a copper IUD poses a transient risk of pelvic inflammatory disease (PID) in the first 21 days after insertion. However, it is a small risk and is attributable to preexisting gonorrhea or chlamydia infection at the time of insertion, and not to the IUD itself. Proper infection prevention procedures have little or no effect on the course of gonorrhea or chlamydia infections, but is important in helping protect both clients and providers from infection in general.

Cramping: Many women feel cramping or pain during the IUD insertion process and immediately after as a result of cervix dilation during insertion. Taking NSAIDS before the procedure can reduce discomfort, as can the use of a local anaesthetic. 
Heavier Periods: The copper IUD increases the amount of blood flow during a woman's menstrual periods. On average, menstrual blood loss increases by $20-50 \%$ after insertion of a copper-T IUD; increased menstrual discomfort is the most common medical reason for IUD removal. This symptom may clear up for some women after 3 to 6 months, but may not for others.

Irregular Bleeding and Spotting: For the first 3 to 6 months after insertion, the copper IUD can cause irregular periods and spotting between periods.

String problems: A small portion of men report that they can feel the strings during intercourse. In this case, strings can be trimmed. However, very short strings can prevent the woman from checking the strings for expulsion. Medical ultrasonography may be required in such cases to check the location of the IUD.

Pregnancy: Although rare, if pregnancy does occur with the copper IUD in place there can be serious side effects. The risk of ectopic pregnancy to a woman using an IUD is lower than the risk of ectopic pregnancy to a woman using no form of birth control.

\section{Contraindications}

Women are pregnant or think they may be pregnant, septic pregnancy or abortion, have unexplained abnormal vaginal bleeding, have untreated cervical cancer, have malignant gestational trophoblastic disease, have uterine cancer, have certain uterine abnormalities, have or may have had a pelvic infection within the past three months, have or may have an STI or have pelvic tuberculosis [8].

According to WHO, Intrauterine device (IUD) with progestogen (shown in Fig. 2): is a hormonal intrauterine device classified as a long-acting reversible contraceptive method. It is one of the most effective forms of birth control. The IUD with progestogen is referred to as an intrauterine system (IUS) or intrauterine contraceptive (IUC). This article will refer to these devices as the hormonal IUD to distinguish from IUD with copper.

\section{Side effects}

\section{Suggested that adverse effects of progestogen IUD} are:

Irregular menstrual pattern: irregular bleeding and spotting is common in the first 3 to 6 months of use. After that time periods become shorter and lighter, and $20 \%$ of women stop having periods after 1 year of use[10].

Cramping and Pain: many women feel discomfort or pain during and immediately after insertion. Some women may have cramping for the first 1-2 weeks after insertion.
Expulsion: Sometimes the IUD can slip out of the uterus. This is termed expulsion. Around 5\% of IUD users experience expulsion.

Perforation: Very rarely, the IUD can be pushed through the wall of the uterus during insertion. Risk of perforation is mostly determined by the skill of the practitioner performing the insertion.

Pregnancy complications: Although the risk of pregnancy with an IUD is very small, if one does occur there is an increased risk of serious problems. These include ectopic pregnancy, infection, miscarriage, and early labor and delivery. As many as half the pregnancies that occur in Mirena users may be ectopic.

Infection: The insertion of the IUD does have a small risk of pelvic inflammatory disease (PID). Concurrent infection with gonorrhea or chlamydia at the time of insertion increases the risk of pelvic inflammatory disease. If PID does occur, it will most likely happen within 21 days of insertion. The device itself does not increase the risk of infection.

Ovarian Cysts: Enlarged follicles (ovarian cysts) have been diagnosed in about $12 \%$ of the subjects using a hormonal IUD. Most of these follicles are asymptomatic, although some may be accompanied by pelvic pain or dyspareunia.

- Mental health changes: Including nervousness, depressed mood, mood swings.

- Weight gain, headache, migraine, nausea, acne, excessive hairiness

- Lower abdominal or back pain, decreased libido, itching, redness or swelling of the vagina [11].

\section{Contraindications}

Women are, or think they may be, pregnant, have abnormal vaginal bleeding that has not been explained, have untreated cervical or uterine cancer, have, or may have, breast cancer, have abnormalities of the cervix or uterus, have had pelvic inflammatory disease within the past 3 months, have had an STI such as chlamydia or gonorrhea within the past 3 months, have liver disease or tumor or have an allergy to levonorgestrel or any of the inactive ingredients included in the device. [12].

\section{SECTION II}

\section{Overview of infection prevention for general clinical} practice

According to Marafie 2012, microorganisms are the causative agents of infection. Microorganisms include bacteria, viruses, fungi, and parasites. In the context of infection prevention, bacteria can be further divided into three categories: vegetative (e.g., staphylococcus), mycobacteria (e.g., tuberculosis), and endospores (e.g., tetanus), which are the most difficult to kill. 
Marafie also added that asepsis or aseptic technique is a general term used to describe the combination of efforts made to prevent entry of microorganisms into any area of the body where they are likely to cause infection. The goal of asepsis is to eliminate, the number of microorganisms on both animate (living) surfaces (e.g., skin and mucous membranes) and inanimate objects (e.g., surgical instruments and other items) [13].

Also Antisepsis is the prevention of infection by killing or inhibiting the growth of microorganisms on skin and other body tissues by using a chemical agent (antiseptic).

Based on [14], protective barriers are physical, mechanical, or chemical processes that help prevent the spread of infectious microorganisms from client to client, clinic staff to client, and client to staff. Also added that infection prevention often relies on placing such barriers between the microorganism and the individual. Examples of protective barriers include: hand washing, wearing gloves, using antiseptic solutions, and processing instruments and other items as described below.

The author agreed with United Nations Development Programme et al. 2013 about infection prevention processes that reduce the number of diseasecausing microorganisms on instruments, gloves, and other items include the following:

Decontamination makes inanimate objects that may have come in contact with blood or other body fluids safer to be handled by staff before cleaning (i.e., reduces, but does not eliminate, the number of microorganisms on inanimate objects).

Cleaning physically removes all visible blood, body fluids, or other material such as dust or dirt from inanimate objects, as well as from skin.

Disinfection eliminates most, but not all, microorganisms from inanimate objects. High-level disinfection (HLD) - by boiling, steaming, or use of chemicals-eliminates almost all microorganisms except some bacterial endospores from inanimate objects.

Sterilization-by autoclaving or dry heateliminates all microorganisms (bacteria, viruses, fungi, and parasites) including all bacterial endospores from inanimate objects.

\section{Standard Precautions}

According to [15], transmission-Based Precautions are infection prevention practices that apply only to hospitalized patients, whereas Standard Precautions are designed for the safety and care of all people in a health care facility — whether a hospitalized patient, a woman receiving IUD services, or a health care worker. Because many people with bloodborne viral infections (e.g., hepatitis B [HBV], HIV) do not feel or look ill, Standard Precautions are to be applied consistently, regardless of the (known or unknown) health status of those who are providing or receiving care. When applied consistently, standard precautions act as protective barriers between microorganisms and individuals, and are considered a highly effective means of preventing the spread of infection. The authors also added with agreement with WHO 2015 that the following considerations and actions help to form such barriers, as well as provide the means for implementing the standard precautions:

Consider every person (client or staff) as potentially infectious and susceptible to infection. Wash hands - the most important procedure for preventing cross- contamination (person to person or contaminated object to person).

In the context of IUD services, hands should be washed before and after conducting the pelvic examination of a potential IUD user, and before and after inserting or removing an IUD.

Wear gloves (on both hands) before touching anything wet - broken skin, mucous membranes, blood or other body fluids (secretions and excretions), soiled instruments, and contaminated waste materials - or for performing invasive procedures. In the context of IUD services, gloves are worn during the pelvic examination of a potential IUD user, and before and after inserting or removing an IUD.

Use physical barriers (protective goggles, face masks, and aprons) if splashes and spills of blood or other body fluids are possible (e.g., when cleaning instruments and other items).

Use antiseptic agents for cleansing skin or mucous membranes before surgery, cleaning wounds, or doing handrubs or surgical handscrubs with an alcohol-based antiseptic product.

Use safe work practices such as not recapping or bending needles, safely passing sharp instruments, and suturing (when appropriate) with blunt needles.

Safely dispose of infectious waste materials to protect those who handle them and prevent injury or spread of infection to the community.

\section{Infection control measures in family planning unit}

The consistent use of recommended infection prevention practices is a critical component of quality health services, as well as a basic right of every patient, client, or staff member in a health care setting. Although there is only a minimal risk of infection associated with IUD use, studies have shown that it is 
often related to the insertion procedure [16], rather than to the IUD itself. When the procedure is performed correctly, however, and in accordance with the recommended infection prevention practices, the rate of infection following IUD insertion is very low-less than $1 \%$. [17]

Key objectives of infection prevention in providing IUD services are to:

- Reduce the risk of infection due to IUD insertion.

- $\quad$ Reduce the risk of disease transmission to IUD clients and potential

- IUD clients.

- Protect health care workers at all levels - from physicians and

- $\quad$ nurses to housekeeping staff-from disease.

\section{SPECIFIC INFECTION PREVENTION TIPS FOR IUD \\ Insertion or removal \\ Appropriate Setting}

According to [18], an examination room in an outpatient clinic or a minor surgery room in a hospital is a suitable setting for IUD insertion or removal. If possible, the room should be located away from heavily used areas of the facility, offer privacy, and:

- Contain an examination or procedure table with a washable surface

- Be adequately lit and well-ventilated (with tight-fitting screens on any open windows).

- Be clean, orderly, and free of dust and insects

- Have tile or concrete floors to facilitate cleaning

- Contain leak-proof containers (with tightfitting lids) or plastic bags for disposal of contaminated waste items.

- Have nearby handwashing facilities, including a supply of clean, running water (i.e., clear, not cloudy or with sediment).

Appropriate Attire for Clients and Staff

Added that because IUD insertion and removal are minor procedures:[18]

- Clients can wear their own clothing, provided it is clean.

- Staff do not have to wear a cap, mask, or gown.

Specific Infection Prevention Measures for the Procedure

According to [19] before IUD Insertion or Removal (as Applicable)

\section{The staff should}

- Ensure that instruments and supplies are available and ready for use.

- Ensure that the IUD package is unopened and undamaged. The IUD package should not be opened until the final decision to insert the IUD has been made.

- Ensure that the woman has wash (with soap and water) and rinse her perineal area.

- Place a dry, clean cloth between her genital area and the surface of the examination table.

- Wash hands thoroughly with soap and water; dry them with a clean, dry cloth or allow them to air dry.

- Put new/clean examination or high-level disinfected (or sterile) surgical gloves on both hands.

\section{During IUD Insertion or Removal (as Applicable) \\ According to [20]:}

Explain the Insertion or removal Procedure

A woman who has chosen the IUD needs to know what will happen during insertion/removal.. Therefore, this description is a summary and not detailed instruction.

- Conduct a pelvic examination to assess eligibility. The provider first does the bimanual examination and then inserts a speculum into the vagina to inspect the cervix.

- Clean the cervix and vagina with appropriate antiseptic.

- Slowly insert the tenaculum through the speculum and closes the tenaculum just enough to gently hold the cervix and uterus steady.

- Slowly and gently pass the uterine sound through the cervix to measure the depth and position of the uterus.

- Load the IUD into the inserter while both are still in the unopened sterile package.

- Slowly and gently insert the IUD and removes the inserter.

- Cut the strings on the IUD, leaving about 3 centimeters hanging out of the cervix.

- After the insertion, the woman rests and remains on the examination table until she feels ready to get dressed.

- Supporting the user

\section{Giving Specific Instructions}

Expect cramping and pain

- She can expect some cramping and pain for a few days after insertion.

- Also, she can expect some bleeding or spotting immediately after insertion. This may continue for 3 to 6 months.

\section{After IUD Insertion or Removal}

Based on [21]before removing gloves the nurse should:

Place all used instruments in $0.5 \%$ chlorine solution for 10 minutes for decontamination, if not already done. 
Dispose of waste materials (e.g., cotton balls) by placing them in a leak-proof container (with tightfitting lid) or plastic bag [22]. Immerse both gloved hands in $0.5 \%$ chlorine solution. Remove gloves by turning them inside out [23].

If disposing of the gloves, place them in the leak-proof container or plastic bag. If reusing the gloves (not recommended), submerge them in $0.5 \%$ chlorine solution for 10 minutes for decontamination [24]. Wash your hands thoroughly with soap and water; dry them with a clean, dry cloth or allow them to air dry [25].

Also agreed with [26] after the client has left, wipe the examination table with $0.5 \%$ chlorine solution to decontaminate.

Ensure that all instruments, gloves, and other reusable items are further-processed according to recommended infection prevention practices [27].

\section{SECTION III \\ Maternity nurses practice of infection control measures}

Studied assessment of health care providers' knowledge and practices of infection control precautions regarding Intra-Uterine Contraceptive Device insertion and removal [27]. Obstetricians and nurses' knowledge about infection control precautions before, during and after IUCD insertion and removal were inadequate $(52.9 \% \& 48.9 \%)$ respectively while obstetricians' and nurses' practices about universal infection control precautions were unsatisfactory $(62.7 \%$ \& $51.1 \%)$ respectively.

Infection control precautions have been strongly recommended by the Center for Disease Control and Prevention (CDC) for many decades as a mean of avoiding the spread of cross-infection, disease outbreaks and to ensure service provider occupational safety [29]. Regarding Obstetricians' knowledge, findings of this study revealed that more than half of Obstetricians and nurses had inadequate knowledge about universal precautions of infection control before, during and after IUD insertion and removal. As regards the universal precautions knowledge, findings of the present study showed that the majority of the nurses and obstetricians had correct answers about knowledge of hand washing techniques before and after IUCD insertion and removal. As regards wearing gloves while dealing with instruments, $(80.0 \%)$ of the nurses used strong gloves. Also, it is clear that about two-thirds of the nurses gave complete correct answers about the proper time of changing gloves, methods of sterilizations, hot air oven and autoclave. As regards Methods of opening IUCD package, about half of all nurses had adequate knowledge of package opening.

Who conducted a study at Assiut, to make an assessment of health team Knowledge and practices about Infection Control in Maternal Child Health Centers in Assiut City, and reported that most health team staff have poor knowledge about hand washing[30]. Defects of Nurses' knowledge about hand washing may be attributed to the fact that they are not concerned with reading updated information; less than half attended conferences, also most nurses in the current study had Diplomas of Nursing degree with a curriculum that has little information about infection control, as mentioned by nurses in the present study the main source of knowledge was conferences and training courses of infection control [31]. Who studied establishing standards for prevention of nosocomial infection in the recovery room and surgical ward at Elminia university Hospital. She mentioned that, although the mean knowledge score in hand washing was fair, their mean practices score was very poor.

These findings of the nurses practices may be attributed to lack of awareness of sufficient time of sterilization, behavior is probably due to a lack of information on infective and non-infective health hazards behaviors in frame work practices, daily high load of patients, insufficient percentage of training courses, lack of awareness of handling and shortage of sterilized instruments, lack of supervision, and careless behaviors.

Who study the discriminatory attitudes and practices by health team toward patients with HIV /AIDS in Nigeria, they found that lack of materials reported by $65 \%$ of professionals and was cited as the main reason for non-practice of universal precautions. Health care providers have cited various reasons for noncompliance including insufficient time, inaccessible supplies and lack of knowledge[32]. Little is known about the factors that motivate them to comply with infection control practices in studying practices and an assessment of health care providers' perception of compliance with infection control care in the new millennium.

\section{CONCLUSION}

This review aims to provide an overview about maternity nurses' practices of infection control measures during insertion and removal of IUDs. This review of literature includes three sections. The first section is an overview about IUD; the second section is devoted to infection control measures in family planning unit while the third explain maternity nurses' practice of such measures.

\section{REFERENCES}

1. Cleland, J., Conde-Agudelo, A., Peterson, H., Ross, J., \& Tsui, A. (2012). Contraception and health. The Lancet, 380(9837), 149-156.

2. Cleland, J., Bernstein, S., Ezeh, A., Faundes, A., Glasier, A., \& Innis, J. (2006). Family planning: the unfinished agenda. The Lancet, 368(9549), 1810-1827. 
3. Rutstein, S. O. (2005). Effects of preceding birth intervals on neonatal, infant and under- five years mortality and nutritional status in developing countries: evidence from the demographic and health surveys. International Journal of Gynecology \& Obstetrics, 89, S7-S24.

4. Kozuki, N., \& Walker, N. (2013). Exploring the association between short/long preceding birth intervals and child mortality: using reference birth interval children of the same mother as comparison. BMC public health, 13(S3), S6.

5. ICPD, U. (1994). Programme of Action of the United Nations International Conference on Population and Development. Cairo, UN.

6. Cohen, S. A., \& Richards, C. L. (1994). The Cairo consensus: population, development and women. Family planning perspectives, 26(6), 272277.

7. Alkema, L., Kantorova, V., Menozzi, C., \& Biddlecom, A. (2013). National, regional, and global rates and trends in contraceptive prevalence and unmet need for family planning between 1990 and 2015: a systematic and comprehensive analysis. The Lancet, 381(9878), 1642-1652.

8. Khatri, R. B., Mishra, S. R., \& Khanal, V. (2017). Female community health volunteers in community-based health programs of Nepal: future perspective. Frontiers in public health, 5, 181.

9. World Health Organization. Department of Mental Health, Substance Abuse, World Health Organization, World Health Organization. Department of Mental Health, Substance Abuse. Mental Health, World Health Organization. Mental Health Evidence, \& Research Team. (2005). Mental health atlas 2005. World Health Organization.

10. MoHP, N. E. (2011). ICF International Inc. Nepal demographic and health survey.

11. Stanton, C., Blanc, A. K., Croft, T., \& Choi, Y. (2007). Skilled care at birth in the developing world: progress to date and strategi es for expanding coverage. Journal of biosocial science, 39(1), 109.

12. MoHP, N. E., \& Macro, O. R. C. (2002). Nepal demographic and health survey 2001. Family Health Division, Ministry of Health, New ERA and ORC Macro, Calverton, Md, USA.

13. Pfitzer, A., Mackenzie, D., Blanchard, H., Hyjazi, Y., Kumar, S., Lisanework Kassa, S., ... \& Zaeem, S. (2015). A facility birth can be the time to start family planning: postpartum intrauterine device experiences from six countries. International Journal of Gynecology \& Obstetrics, 130, S54-S61.

14. Kapp, N., \& Curtis, K. M. (2009). Intrauterine device insertion during the postpartum period: a systematic review. Contraception, 80(4), 327-336.

15. World Health Organization. (2013). Programming strategies for postpartum family planning.

16. Lopez, L. M., Bernholc, A., Hubacher, D., Stuart, G., \& Van Vliet, H. A. (2015). Immediate postpartum insertion of intrauterine device for contraception. Cochrane Database of Systematic Reviews, (6).

17. McPake, B., Witter, S., Ensor, T., Fustukian, S., Newlands, D., Martineau, T., \& Chirwa, Y. (2013). Removing financial barriers to access reproductive, maternal and newborn health services: the challenges and policy implications for human resources for health. Human resources for health, 11(1), 46.

18. Karra, M., Canning, D., Foster, S., Shah, I. H., Senanayake, H., Ratnasiri, U. D. P., \& Pathiraja, R. P. (2017). Location and content of counselling and acceptance of postpartum IUD in Sri Lanka. Reproductive health, 14(1), 42.

19. Canning, D., Shah, I. H., Pearson, E., Pradhan, E., Karra, M., Senderowicz, L., ... \& Langer, A. (2016). Institutionalizing postpartum intrauterine device (IUD) services in Sri Lanka, Tanzania, and Nepal: study protocol for a cluster-randomized stepped-wedge trial. BMC pregnancy and childbirth, 16(1), 362.

20. Clarke, P. S., Palmer, T. M., \& Windmeijer, F. (2015). Estimating structural mean models with multiple instrumental variables using the generalised method of moments. Statistical Science, 96-117.

21. Hernán, M. A., \& Hernández-Díaz, S. (2012). Beyond the intention-to-treat in comparative effectiveness research. Clinical trials, 9(1), 48-55.

22. Bertrand, M., Duflo, E., \& Mullainathan, S. (2004). How much should we trust differences-indifferences estimates?. The Quarterly journal of economics, 119(1), 249-275.

23. Cameron, A. C., Gelbach, J. B., \& Miller, D. L. (2008). Bootstrap-based improvements for inference with clustered errors. The Review of Economics and Statistics, 90(3), 414-427.

24. Webb, M.D. (2014). Reworking wild bootstrap based inference for clustered errors [internet]. Queen's University, Department of Economics; 2014 [cited 2016 Dec 16]. Report No.: 1315. Available from: https://ideas.repec.org/p/qed/ wpaper/1315.html.

25. Roodman, D. (2016). BOOTTEST: Stata module to provide fast execution of the wild bootstrap with null imposed [Internet]. Boston College Department of Economics; 2016 [cited 2016 Dec 16]. (Statistical Software Components). Available from: https://ideas.repec.org/c/boc/bocode/s458121.html

26. Makins, A., Taghinejadi, N., Sethi, M., Machiyama, K., Munganyizi, P., Odongo, E., ... \& Arulkumaran, S. (2018). FIGO postpartum intrauterine device initiative: Complication rates across six countries. International Journal of Gynecology \& Obstetrics, 143, 20-27.

27. Puri, M. C., Maharjan, M., Pearson, E., Pradhan, E., Dhungel, Y., Khadka, A., \& Shah, I. H. (2018). Delivering postpartum family planning services in 
Nepal: are providers supportive?. BMC health services research, 18(1), 1-9.

28. Lith, L.M.V., Yahner, M., Bakamjian, L. (2013). Assessment of health care providers' knowledge and practices of infection control precautions regarding Intra-Uterine Contraceptive Device insertion and removal. Glob Health Sci Pract, 1(1):97-107.

29. Shah, I. H., \& Åhman, E. (2012). Unsafe abortion differentials in 2008 by age and developing country region: high burden among young women. Reproductive health matters, 20(39), 169173.

30. Darroch, J.E., Woog, V., Bankole, A., Ashford, L. (2016). Assessment of health team Knowledge and practices about Infection Control in Maternal Child Health Centers in Assiut City. [Internet].
Guttmacher Institute; [cited 2016 Dec 21]. Available from: http://www.popline.org/ node/649435

31. Singh, S., Darroch, J.E. (2012). Establishing standards for prevention of nosocomial infection in the recovery room and surgical ward at Elminia university Hospital. Estimates for, 2012; Guttmacher Institute, United Nations Population Fund: New

York http://www.guttmacher.org/pubs/AIU2012- estimates.pdf. Accessed April 27, 2014

32. Cleland, J., Conde- Agudelo, A., Peterson, H., Ross, J., Tsui, A. (2012). discriminatory attitudes and practices by health team toward patients with HIV /AIDS in Nigeria. Lancet, 380(9837): 149-150. 